\title{
Obstacle Detection from IPM and Super-Homography
}

\author{
Nicolas SIMOND \\ IMARA project-team INRIA Paris-Rocquencourt \\ Domaine de Voluceau, 78153 Le Chesnay Cedex, France \\ Nicolas.Simondeinria.fr
}

\begin{abstract}
We present in this article a simple method to estimate an IPM view from an embedded camera. The method is based on the tracking of the road markers assuming that the road is locally planar. Our aim is the development of a freespace estimator which can be implemented in an Autonomous Guided Vehicle to allow a safe path planning. Opposite to most of the obstacle detection methods which make assumptions on the shape or height of the obstacles, all the scene elements above the road plane (particularly kerbs and poles) have to be detected as obstacles. Combined with the IPM tranformation, this obstacle detection stage can be viewed as the first stage of a free-space estimator dedicated to AGV in the complex urban environments.
\end{abstract}

\section{INTRODUCTION}

\section{A. Related works}

For two years now, most of the automotive manufacturers propose on their new cars Advanced Driving Assistance Systems that allow active security like Automatic Cruise Control, Lane Crossing Detection, obstacle avoidance, etc. The obstacle detection systems can be divided into different groups according to the types of obstacle the system detects, the ranges, the refresh rate, the reliability.

The range-finders based on radars, Laser or Lidar provide high spatial resolutions data at high scanning speeds for long ranges but remain too much expensive for automotive applications. Furthermore, the interpretation of the beam impacts on the environment is difficult in dynamic and complex environments. Cheaper sensors like sonars are nevertheless used for difficult maneuver as parking but are useless at higher speed. The drawbacks of range-finder methods are improved as soon as they are coupled with a vision system to perform a segmentation of the scene.

The vision sensor appears particularly well adapted to segment complex scenes thanks to the rich information it captures in a single frame. Several methods have been implementing for years now: they essentially differ according to the applications. From highways to urban environment, many factors vary like the density of traffic, the field of view, the types of obstacles (vehicles, trucks, cycles, pedestrians, others), the numbers of road lanes, the presence or not of road markers. In [1], where an exhaustive recent review of the vehicle detection is presented, all the methods are visionbased except those that are coupled with a range finder.

Methods using a mono camera are generally based on apriory knowledge of the scene and particularly on the type of obstacles. These methods are dedicated to detect either vehicles according their vertical edges, shadows, symmetry, lights [2] or pedestrians and crowd according to human shape and behaviour constraints. Some original methods track sets of features points ([3]) or edges ([4]) on obstacles because their relative motion is different from the camera motion. In a general way, the segmentation of the elements of a scene can be performed with a dense estimation of the optical flow [5] and the introduction of colour, texture constraints ([6]) or a parametrization of the static world [7].

An other technique common to mono and stereo-vision consist on considering all the elements which are lying above the road as obstacles. In a first step, the projection of the road surface is segmented according to a-priory knowledges (road model, planarity assumption). The estimation of camera motion between two poses (relative motion of the static road) in a second step, allows, in a third step, the detection of obstacles according to their elevations which don't verify the road surface motion (optical flow) or they are distorted (homography).

The stereo-vision allows by triangulation the estimation of the depth of homologous pixels matched in a pair of images if the stereo-rig is calibrated. When the images are rectified, the computation of the V-disparity, an original method developed by Labayrade in [8], allows with real-time constraints the segmentation of obstacles whatever the longitudinal profile of the road and the discrimination between vehicles and trucks. Many authors perform the method with introducing U-disparity [9], infrared sensors to detect pedestrians [10] or multi-baseline sensors for off-road environment [11].

\section{B. Motivations}

The method we present in this paper is dedicated to Autonomous Guided Vehicles where the estimation of the free-space in front of the mobile robot is more crucial than the identification of the obstacles. In other words, all the elements which are lying above the road surface represent an obstacle, whatever their shape or height. Hence, the kerbs and the green strips which generally highlight the roads boundaries have to be segmented as obstacles if they have a non-null elevation.

Our aim is also the detection of all the obstacles which reduce the free-space to allow an overtaking maneuver if it is necessary. This study represents the last improvement of a feature-based method we have been developing for some years ([12]) to locate a mobile robot when GPS data are corrupted. The method is inspired by the works of Okutomi [13], which proposed to identify the road surface 
with the homography induced in images captured by a calibrated stereo-rig. The computation is performed with a dense method whereas we use points and lines extracted with a Harris and a Canny detector.

The main restriction of the method remains on the assumption of flat road that seems not so restrictive in real conditions due to the limited field of view in case of urban traffic. We hence assume that the free space in front of the vehicle does not exceed some meters, that corresponds to the security distance with the preceding vehicle. Nevertheless, the proposed method has the significant interest not to depend to the calibration of the stereo-rig.

This paper is organized as follows: in Section II, we propose a method to compute easily a bird eye view of the road plane. The expression in the IPM image of the features lying above the road plane is presented in section III. Some results obtained on video sequences are shown in Section IV. The conclusion and a list of possible research directions are given in Section $\mathrm{V}$.

\section{INVERSE PERSPECTIVE MAPPING}

\section{A. Interest}

We present in this part a simple methodology to compute an Inverse Perspective Mapping (IPM) image [14] or a Virtual Projection Plane, as it is called by some authors. The IPM transformation consist on modifying the angle of view under which a scene is acquired to remove the perspective effect. In a practical way, the IPM transformation consist to set the angles of rotation (mainly the tilt one) to values such as the normal to the plane $\mathbf{n}$ and the focal axis of the virtual camera $\mathbf{z}_{\mathbf{v}}$ are opposite: $\mathbf{n} . \mathbf{z}_{\mathbf{v}}=-1$, according to the Fig. 1. The virtual camera follows the vehicle path but has a constant ordinate $z_{v}$ along its $\mathbf{z}$ axis, whatever the slope of the road $s$.

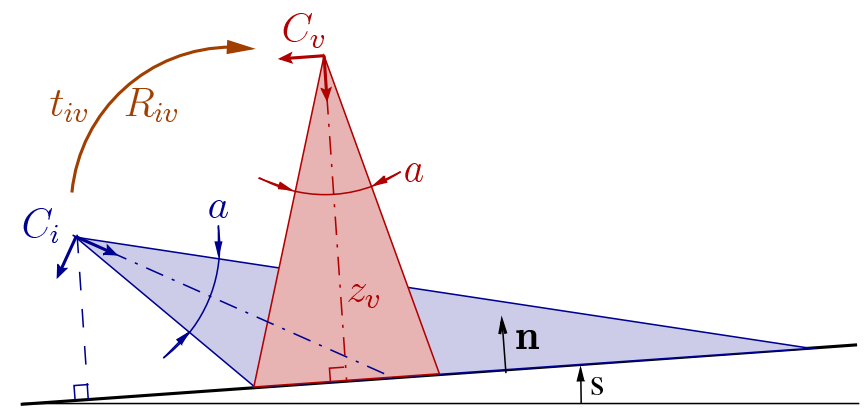

Fig. 1. Principle of the IPM transformation: the virtual camera $\mathcal{R}_{v}$ has a constant elevation $z_{v}$ along the third $\mathbf{z}_{\mathbf{v}}$ axis which is parallel to the normal to the plane $\mathbf{n}$ whatever the camera pose $\mathcal{R}_{i}$ and the road slope $s$ while the aperture angle $\alpha$ is maintained.

The method only requires the extraction of two coplanar parallel lines. Due to the perspective effect, the 3D lines which are parallel have projections in image which converge to a Vanishing Point. The IPM transformation also consist on determining the point of view of a virtual camera where the projections of the two lines appear parallel.

In case of structured road, the navigation lanes are usually highlighted with parallel painted markers. Hence, the extraction of two parallel lines along a straight path is relatively easy. Many authors propose several models of the road projection that mainly differ on the free field of view, the presence of road markers or not, the number of degrees of liberty the road model assumes. We personally use the simplest model: the road borders are linearized at the foreground and converge to the Dominant Vanishing Point (DVP) $\mathbf{x}$ whatever the road curvature is. The reader can retrieve in [15] the tracking process we have developed to segment the projection of the road in images.

In the following, we detail the method to compute an IPM while in the second part, we discuss on the interest of the method.

\section{B. Methodology}

Lets now considering $\mathcal{R}_{i}$ and $\mathcal{R}_{v}$ the frameworks relative to the camera and the virtual camera. We assume in the following that the original and virtual images have the same dimensions $[W, L]$ and 256 gray levels.

We also introduce two horizontal lines $\mathbf{l}_{\mathbf{t}}$ and $\mathbf{l}_{\mathbf{b}}$ which have respectively $v_{t}$ and $v_{b}$ v-ordinates. In the original image, the intersections of these two lines with the pencil of road markers (restricted to the first and the last median) and the image vertical boundaries generate two quadruplets of points. The first one $\mathbf{p}_{\pi \mathbf{i k}}$ with $k \in\{1,2,3,4\}$ forms a trapezoid, as in Fig. 2, while the second one $\mathbf{p}_{\text {eik }}$ which is the bottom part of the original image forms a rectangle.

If the road has a constant width in the scene, its projection in the virtual framework is also constant $w_{\pi v 0}$, that means the four points $\mathbf{p}_{\pi \mathbf{v k}}$ form a parallelogram in $\mathcal{R}_{v}$ whereas the image is represented with a truncated cone. The IPM transformation can hence be resumed as a homography $\mathrm{H}_{\mathrm{vi}}$ such as:

$$
\begin{aligned}
& \mathbf{p}_{\pi \mathrm{vk}} \simeq \mathrm{H}_{\mathrm{vi}} \mathbf{p}_{\pi \mathrm{ik}} \\
& \mathbf{p}_{\text {evk }} \simeq \mathrm{H}_{\mathrm{vi}} \mathbf{p}_{\text {eik }}
\end{aligned}
$$

where $\simeq$ represents a projective equality.

1) Initialization of the IPM process: At this point, the coordinates of the two quadruplets in the virtual framework $\mathcal{R}_{v}$ are unknown. We only suppose that the aperture angle of the camera lens $\alpha$ is supposed to be known and the elevation $z_{v}$ of the virtual camera relative to the plane is constant. That means if we fixed the location of the cone summit c, the projection of the original image in $\mathcal{R}_{v}$ is invariant whatever the camera pose in $\mathcal{R}_{i}$. To maintain a coherence in the projective transformation, we consider that the top and the bottom lines are invariant:

$$
\begin{aligned}
l_{\mathrm{bv}} & \simeq \mathrm{H}_{\mathrm{vi}}^{-\mathrm{t}} \mathrm{l}_{\mathrm{bi}} \simeq \mathrm{l}_{\mathrm{b}} \\
\mathrm{l}_{\mathrm{tv}} & \simeq \mathrm{H}_{\mathrm{vi}}^{-\mathrm{t}} \mathrm{l}_{\mathrm{ti}} \simeq \mathrm{l}_{\mathrm{t}}
\end{aligned}
$$

The quadruplet $\mathbf{p}_{\text {evk }}$ also forms an isosceles trapezoid whose the little base is supported by $l_{b}$ and the vertical axis represents a symmetry axis.

In an other hand, the cross-ratio and the incidence are the lonely properties left invariant by a projective transformation. 


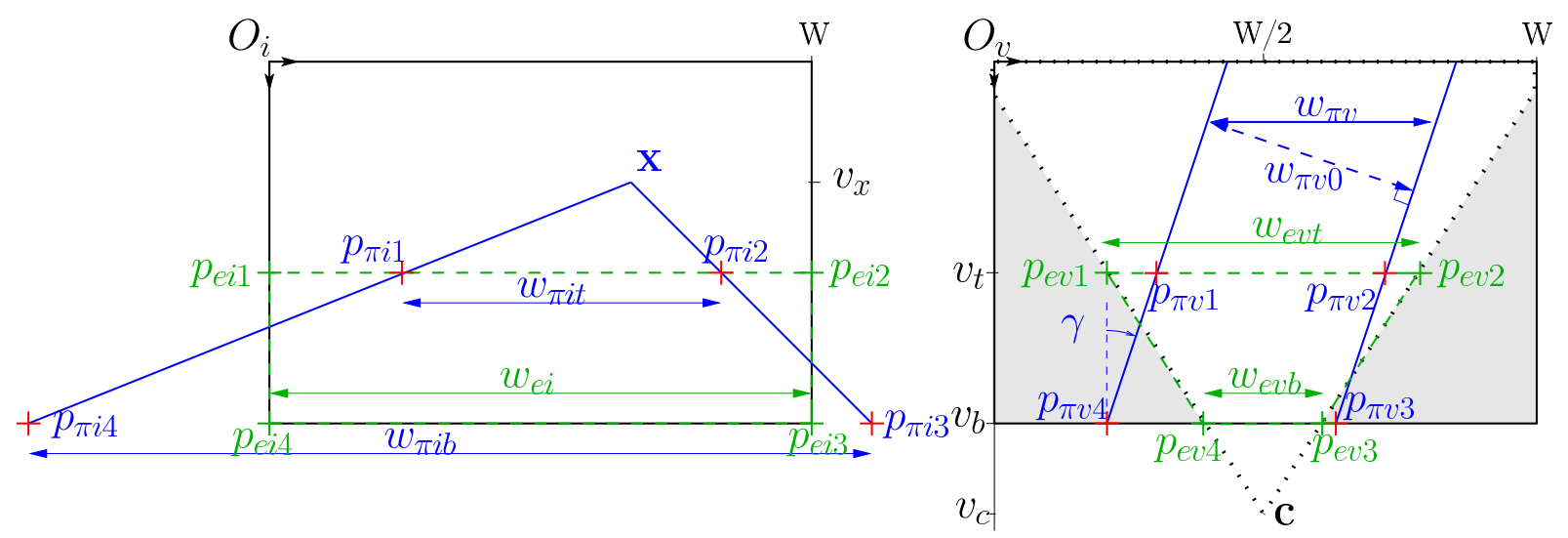

Fig. 2. Principle of the IPM transformation: the road boundaries converge to the DVP $\mathbf{x}$ in $\mathcal{R}_{i}$ and appear parallel in $\mathcal{R}_{v}$. The intersections of the image boundaries and the road borders with the two parallel lines $\mathbf{l}_{\mathbf{b}}$ and $\mathbf{l}_{\mathbf{t}}$ respectively generate the quadruplets $\left\{\mathbf{p}_{\mathbf{e} 1}, \mathbf{p}_{\mathbf{e} 2}, \mathbf{p}_{\mathbf{e} 3}, \mathbf{p}_{\mathbf{e} 4}\right\}$ and $\left\{\mathbf{p}_{\pi 1}, \mathbf{p}_{\pi 2}, \mathbf{p}_{\pi 3}, \mathbf{p}_{\pi 4}\right\}$.

For the two quadruplets of points, the consistency of the cross-ratio is checked ${ }^{1}$ :

$$
\begin{aligned}
& C R\left(\mathbf{p}_{\mathrm{ei} 1}, \mathbf{p}_{\mathrm{ri} 1}, \mathbf{p}_{\mathrm{ri} 2}, \mathbf{p}_{\mathrm{ei} 2}\right)=C R\left(\mathbf{p}_{\mathrm{ev} \mathbf{1}}, \mathbf{p}_{\mathrm{rv} \mathbf{1}}, \mathbf{p}_{\mathrm{rv} \mathbf{2}}, \mathbf{p}_{\mathrm{ev} \mathbf{2}}\right)(5) \\
& C R\left(\mathbf{p}_{\mathrm{ei} 4}, \mathbf{p}_{\mathrm{ri} 4}, \mathbf{p}_{\mathrm{ri} 3}, \mathbf{p}_{\mathrm{ei} 3}\right)=C R\left(\mathbf{p}_{\mathrm{ev} \mathbf{4}}, \mathbf{p}_{\mathrm{rv} \mathbf{4}}, \mathbf{p}_{\mathrm{rv} \mathbf{3}}, \mathbf{p}_{\mathrm{ev} \mathbf{3}}\right)(6)
\end{aligned}
$$

To entirely constraint the system form with the two quadruplets $\mathbf{p}_{\pi \mathbf{v k}}$ and $\mathbf{p}_{\text {evk }}$, we now have to determine the v-ordinates $v_{c}$ of the cone summit (the u-ordinate is assumed fixed $u_{c}=W / 2$ ) and $v_{b}$ of the line $\mathbf{l}_{\mathbf{b}}$.

While there exist an infinity of solutions to the IPM problem, a trivial solution consist to fix the width of the plane projection $w_{\pi v 0}$ that determines the elevation $z_{v}$ of the virtual camera and induces the v-ordinate $v_{c}$ of the cone summit. At this point, the pan angle $\gamma$ between the camera axis and the road direction is unknown. Therefore, we personally prefer to fix $w_{\pi v}=200$ pixels to compute the locations of $\mathbf{c}, \mathbf{p}_{\text {ev } 3}, \mathbf{p}_{\text {ev } 4}$. The width of the little base $w_{e b}$ of the trapezoid is also computed thanks to the consistency of the cross-ratio (6): the points $\mathbf{p}_{\text {ev } 4}, \mathbf{p}_{\text {ev3 }}$ have invariant locations and form an isosceles triangle with the cone summit $\mathbf{c}$. The v-ordinate $v_{c}$ is such as:

$$
v_{c}=v_{b}+\frac{w_{e b} / 2}{\tan (\text { alpha } / 2)}
$$

where $v_{b}=L$ is the height of the virtual image. The width of the great base $w_{e t}$ is computed thanks to the consistency of the cross-ratio (5), according to:

$$
w_{\pi v}=w_{\pi v b}=w_{\pi v t}=\overline{\mathbf{p}_{\pi \mathbf{v} \mathbf{3}}-\mathbf{p}_{\pi \mathbf{v} \mathbf{4}}}=\overline{\mathbf{p}_{\pi \mathbf{v} \mathbf{2}}-\mathbf{p}_{\pi \mathbf{v} \mathbf{1}}}
$$

The v-ordinate $v_{t}$ of the top line $\mathbf{l}_{\mathbf{t}}$ can also be computed as:

$$
v_{t}=\frac{w_{\pi v} / 2}{\tan (\operatorname{alph} a / 2)}-v_{b}
$$

and the road width $w_{\pi v 0}$ is linked to the camera orientation $\gamma$ as:

$$
w_{\pi v 0}=w_{\pi v} \cos (\gamma)
$$

\footnotetext{
${ }^{1}$ Whatever the quadruplet of aligned points $\{A, B, C, D\}$, $C R(A, B, C, D)=\frac{\overline{B-A}}{\overline{C-B}} / \frac{\overline{D-A}}{\overline{C-D}}=c t e$
}

with $\overline{\mathbf{p}_{\pi \mathbf{v} \mathbf{1}}-\mathbf{p}_{\pi \mathbf{v} \mathbf{4}}}=\left(v_{b}-v_{t}\right) \tan (\gamma)$.

The homography $\mathrm{H}_{\mathrm{vi}}$ is now totally defined thanks to the quadruplet $\mathbf{p}_{\text {eik }}$ and their image $\mathbf{p}_{\text {evk }}$. We show in Fig. 3 an IPM image computed with this procedure. Although the camera is not calibrated, we compute up to a scale factor the orientation $\gamma$ and the lateral position relatively to the road direction and width. We propose on our web site ${ }^{2}$ the result of this transformation along a video sequence where the pan and tilt rotation motions of the camera vary within large ranges.

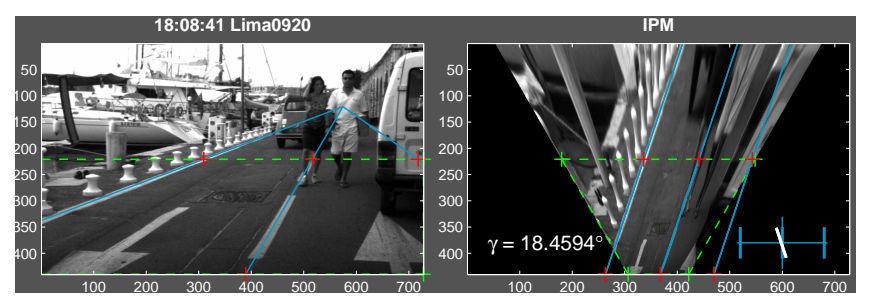

Fig. 3. The IPM transformation is computed thanks to the intersections of the first and the third blue medians of the road boundaries with the green $\mathbf{l}_{\mathbf{b}}$ and $\mathbf{l}_{\mathbf{t}}$ lines. The second median is shown for convenience.

2) Simplifications: The consistency of the cross-ratio is still checked if an affine transformation is applied to the quadruplet. For the lines $\mathbf{l}_{b}$ and $\mathbf{l}_{\mathbf{t}}$, the IPM transformation corresponds to a translation with a scale factor. To compute $w_{e b}$ then $w_{t b}$, we had better to check directly the following ratio rather than use (5) and (6), which lead to quadratic equation where we have to determine the right solution:

$$
\frac{w_{\pi i}}{w_{e i}}=\frac{w_{\pi v}}{w_{e v}}
$$

where $w_{e i}=W$, the image width.

As soon as the location of the optical axis of the virtual camera $\mathbf{c}$ is fixed, the determination of the top v-ordinate $v_{t}$ highly depends on the location of the DVP $\mathbf{x}$. The Thales theorem applied to the triangles $\left\{\mathbf{x}, \mathbf{p}_{\pi \mathbf{i} 4}, \mathbf{p}_{\pi \mathbf{i} 3}\right\}$ and

\footnotetext{
${ }^{2}$ http://www.lara.prd.fr/Nicolas.Simond
} 
$\left\{\mathbf{c}, \mathbf{p}_{\mathbf{e v} \mathbf{1}}, \mathbf{p}_{\mathbf{e v} \mathbf{2}}\right\}$, crossed by the parallel lines $\mathbf{l}_{\mathbf{b}}$ and $\mathrm{l}_{\mathbf{t}}$, allows the following equalities:

$$
\begin{aligned}
\frac{w_{\pi i t}}{w_{\pi i b}} & =\frac{v_{t}-v_{x}}{v_{b}-v_{x}} \\
\frac{w_{e v b}}{w_{e v t}} & =\frac{v_{c}-v_{t}}{v_{c}-v_{b}}
\end{aligned}
$$

According to $v_{c}, v_{b}, w_{e v b}$ are now fixed and $w_{\pi i b}, v_{x}$ are measured in the original image. The other variables $w_{\pi i t}, w_{\text {evt }}$ depend on the $v_{t}$ ordinate. Parallely, a new equality can be introduce from (8) and (11):

$$
\frac{w_{\pi i t}}{w_{\pi i b}}=\frac{w_{\pi i t}}{w_{e i t}} \cdot \frac{w_{e i b}}{w_{\pi i b}}=\frac{w_{\pi v t}}{w_{e v t}} \cdot \frac{w_{e v b}}{w_{\pi v b}}=\frac{w_{e v b}}{w_{e v t}}
$$

The introduction of (12) in the last equation implies:

$$
\frac{v_{t}-v_{x}}{v_{b}-v_{x}}=\frac{v_{c}-v_{t}}{v_{c}-v_{b}}
$$

Hence, the v-ordinate of the $\mathbf{l}_{\mathbf{t}}$ line originally fixed with (9) becomes:

$$
v_{t}=v_{c}+v_{x}-v_{b} .
$$

\section{Discussion}

The main drawback of the method is that the IPM image doesn't have squared pixels. The method is simple and only requires the aperture angle of the camera lens. But due to the perspective projection, the bottom line $\mathbf{l}_{\mathbf{b}}$ doesn't have a projection in $\mathcal{R}_{v}$ on the same v-ordinate $v_{t}$ but rather some pixels above. The trouble is we can't estimate how many. Therefore a calibration stage is necessary to determine the two different scale factors which have to be applied on each axis to authorize metric measures.

As soon as two coplanar parallel lines can be detected, the method provides a solution to the IPM transformation. In the other cases, the IPM transformation can be computed thanks to the estimation of the camera motion from the last pose where the IPM was estimated. Lets consider $\mathrm{H}_{\mathrm{vi}}\left(\mathrm{k}_{0}\right)$ the last IPM transformation available at the frame $k=k_{0}$. At the frame $k=k_{0}+N$, the camera motion can be computed from the camera pose at $k=k_{0}$ as the sum of the $N$ elementary motions between each camera pose:

$$
\mathrm{H}_{\mathrm{k}_{0}+\mathrm{N}, \mathrm{k}_{0}} \simeq \mathrm{H}_{\mathrm{k}_{0}+\mathrm{N}, \mathrm{k}_{0}+\mathrm{N}-1} \cdots \mathrm{H}_{\mathrm{k}_{0}+2, \mathrm{k}_{0}+1} \cdot \mathrm{H}_{\mathrm{k}_{0}+1, \mathrm{k}_{0}}
$$

The IPM transformation at the frame $k=k_{0}+N$ is also computed as:

$$
\mathrm{H}_{\mathrm{vi}}\left(\mathrm{k}_{0}+\mathrm{N}\right) \simeq \mathrm{H}_{\mathrm{k}_{0}+\mathrm{N}, \mathrm{k}_{0}} \cdot \mathrm{H}_{\mathrm{vi}}\left(\mathrm{k}_{0}\right) \cdot \mathrm{H}_{\mathrm{k}_{0}+\mathrm{N}, \mathrm{k}_{0}}^{-1}
$$

Thanks to the computation of the Super-Homography (cf. [15]), we can afford to reconstruct the road plane with a mosaicing of warped images all expressed in the first one. The reference image was a bird-eye view of the first image of the video sequence of 312 frames long that corresponds to a path greater than $250 \mathrm{~m}$. We hence would like to stress that if the vehicle path reach an unstructured road or an intersection, the loss of the two parallel coplanar lines during a short period is not crucial.

\section{OBSTACLE DETECTION THANKS TO THE SUPER-HOMOGRAPHY}

\section{A. Methodology}

The accuracy of the homographies extracted from the computation of the Super-Homography leads us to detect all the elements which are above the considered plane. Thanks to the feature-based method we have developed, which identifies coplanar homologous lines and points matched in several views of the same plane, we had the choice to detect the obstacles with points and/or lines. We nevertheless use edges because the matching process for points correlation is time consuming and the obstacles have sometimes uniform textures.

We present in this section a two-stages method where the coplanar edges are first identified to be removed, then the remaining edges are projected with an IPM transformation to determine the regions free from obstacles. We also only focus on edges which have at least one extremity under the horizon line, assumed as the line which has the same vordinate as the DVP.

\section{B. Identification of edges above the horizon line}

The edges which are crossing the horizon line have projections in the IPM which are passing through the projection center c of the virtual camera. With the real data all the vertical edges don't converge to the same point but they generally have extremities out of the image boundaries as in Fig. 4. The identification of the edges above the horizon line is also straight forward thanks to the IPM transformation we detailed in the last section.

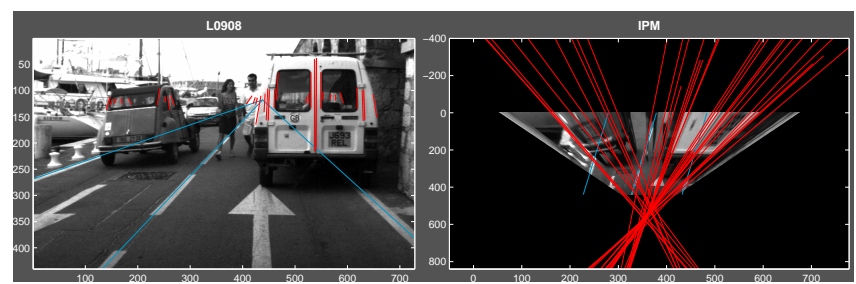

Fig. 4. Identification with an IPM transformation of the edges whose extremities are above and under the horizon line.

\section{Identification of coplanar edges with homography}

Lets considering two views of a same planar scene and $\mathrm{H}_{21}$ the homography induced by the plane between the images $\mathcal{I}_{1}$ and $\mathcal{I}_{2}$. The projection according $\mathrm{H}_{21}$ of the features of one image into the other one allows the identification of the coplanar features because only the coplanar features are overlapping with their homologous. The main difficulty we also face is the edges detected with a Canny detector are not strictly overlapping. A 3D edge can be plotted with a long segment in one image and with zero, one, two or more in the other.

The matching of edges between two images thanks to the homography $\mathrm{H}_{21}$ can be nevertheless performed with a two steps process. The pre-selection stage consist on assuming as potential homologous the couples of edges whose the 


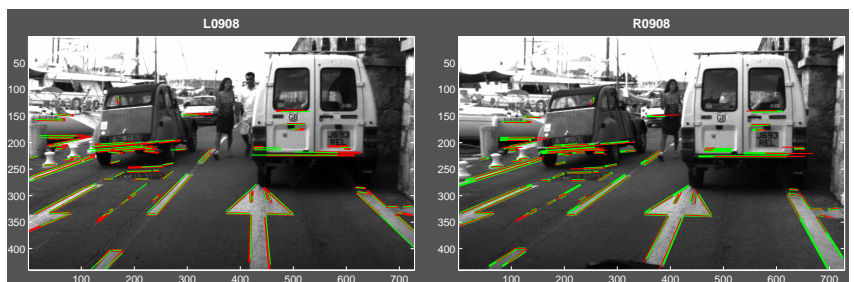

(a) Identification of coplanar edges which are overlapping by retro-projection between the left and right images.

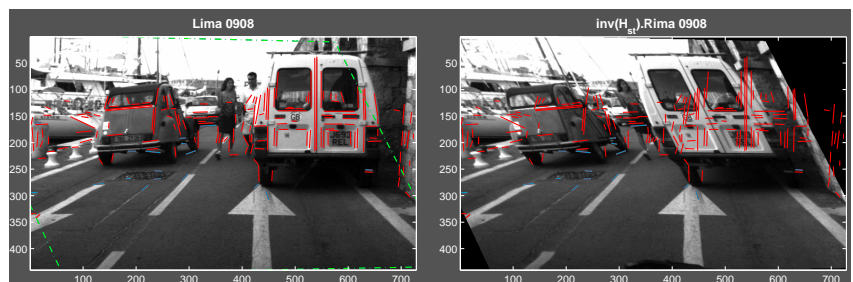

(b) Identification of coplanar edges (blue) which have similar neighborhood in original and warped images.

Fig. 5. Sequential search of the coplanar edges in the left (red) image thanks to the right (green) image and the stereo homography $\mathrm{H}_{\mathrm{st}}$.

extremities, computed by retro-projection, are close to their homologous with a tolerance of 3 pixels. The pre-selection is checked for edges of $\mathcal{I}_{1}$ in $\mathcal{I}_{2}$ and inversely with $\mathrm{H}_{21}^{-1}$. As soon as the couples have been identified, the selection stage consists on verifying the planarity assumption with checking if the couple of edges have common u- or v-ordinates when they are expressed in the same image.

The edges, shown in Fig. 5(a), issued from the left (red) and right (green) cameras, check the overlapping criterion. As expected, the majority of outliers (above the road plane) are horizontal edges whose the pan angle between the camera framework is not large enough to discriminate them with such a method whatever the camera motion. The best way is certainly do not take account the horizontal edges because the determination of their coplanarity is not reliable.

We actually obtain similar results if we only consider the camera motion between two consecutive poses. The trouble is the rate of ouliers increases when the camera motion is not large enough. With such a configuration, the camera frame-rate should be synchronized with the vehicle speed to maintain a critical change of view. The best method certainly consists on tracking the coplanar edges with two couples of views: stereo and consecutive. But as far as we are concerned, the results provided by the stereo-images are sufficiently right to allow a reliable discrimination even some coplanar edges can't be be segmented as if when they are occluded by an obstacle in one image.

\section{Identification of coplanar edges with correlation}

The last sequential operations allow the detection of the majority of the coplanar edges. The left coplanar edges don't fit precisely the contours or don't have homologous in the second view due to occlusion or illumination effects. The rejection of these edges requires one additional process to verify their planarity. Generally, Sum of Absolute Difference (SAD) image computed from the difference between the warping of one image into an other according to a transformation, highlight the scene regions where the assumption (planarity, same relative motion) is checked because their gray levels are the darkest.

The discrimination between edges lying on or above the road with SAD image is not easy due to the illumination conditions the areas close to the edges often appear clearest, whatever the quality of the estimation of homography. Therefore we prefer using normalized criteria on 3 pixels large region centered on each remaining edge in the original and warped images. We fix two new criteria based on the correlation coefficient to consider edges as coplanar: it has to be either greater than 0.75 or greater than 0 with a variance less than 400 and the meaning gray level have to be less than 24 for a 256 gray levels image.

Although some edges have no homologous in the other image, all the (blue) coplanar edges are rejected with the application of the correlation criterion. The (red) remaining edges of the Fig. 5(b) have to be considered as lying above the road plane.

\section{RESULTS}

\section{A. Camera rotation motions estimated with IPM}

The decomposition of the homography $\mathrm{H}_{\pi v i}$ requires the knowledge of the internal parameters of the camera $\mathrm{K}$, an estimation of the normal to plane $\mathbf{n}=[0 ;-1 ; 0]^{t}$ (close to the vertical axis) and an estimation of the camera elevation $d$ in the absolute framework, linked to the road plane. A coarse calibration of the stereo-rig allows us to estimate the three rotation $R$ and translation $\mathbf{t}$ motions required to compute the IPM image and the evolution of the normal of the plane $\mathbf{n}$ along the vehicle path according to:

$$
\mathrm{H}_{\pi \mathrm{vi}}=\mathrm{K}\left[\mathrm{R}+\frac{\mathbf{t} \cdot \mathbf{n}^{\mathbf{t}}}{\mathrm{d}}\right] \mathrm{K}^{-1}
$$

During the Antibes sequence, the test-vehicle follows a straight path, stops before a parked vehicle, overtakes it then continues its straight motion. We present the decompositions of the IPM homography in Fig. 6. The smoothness of the different time diagrams is relevant: the IPM homography is only computed with four couples of matched points. The numerical values of the rotation motions are not correct: assuming that the optical axis $\mathbf{z}$ is abroad $20^{\circ}$ under the horizon line, we expect a rotation around the $\mathbf{x}$ axis higher than $-17^{\circ}$. Such an error is mainly due to the scale factors we introduce with our coarse calibration. The decomposition of the translation motions seem reliable: to obtain an IPM view, the translation is mainly support by the $\mathbf{z}$ and in a second time by the $\mathbf{y}$, as in Fig. 1 .

\section{B. Free-space segmentation}

In the preceding section, we explain how to discriminate between edges lying on or above a plane. The best way to interpret this result is using an IPM transformation to produce 

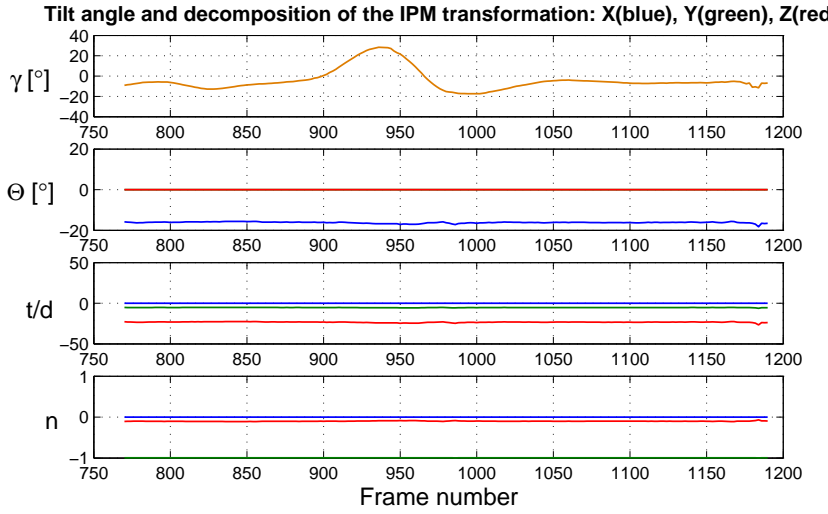

Fig. 6. Orientation of the camera (brown) and decomposition of the $\mathrm{H}_{\pi v i}$ homography along the Antibes sequence. The numerical values are given up to a scale factor due to the pixels of the IPM image are not squared and the camera is not calibrated $(d=1 \mathrm{~m})$.

a polar chart of the obstacle edges. We can hence segment in the 2D scene the areas which are free from obstacles in front of the vehicle thanks to a bird-eye view. The constant constraints (road width and aperture angle) on IPM images allow a correct segmentation of the obstacles in the road framework at two scale-factors due to the calibration of the stereo-rig is not available.

Opposite to most of the methods developed on the reconstruction of the 3D scene based on (V-)disparity, the proposed method does not make assumption on the obstacles elevation. The limitation of the method is the length of the edges which have to be greater than 10 pixels, that allows us to detect the majority of the white small bollards which are lying on left road boundary of the Antibes sequence like in Fig. 7. That is our goal for an implementation in an AGV.

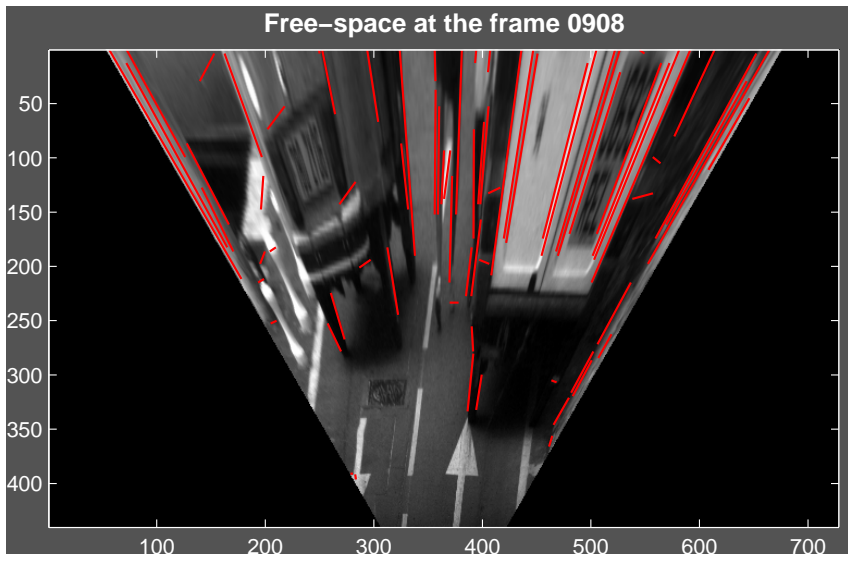

Fig. 7. The free-space in front of the camera is the lowest region of the IPM image under the obstacles edges. The edges which are crossing the horizon line are removed according to the methodology developed in III-B.

\section{CONCLUSiOns AND Future Works}

We present in this paper a methodology to compute reliable IPM transformation as soon as two parallel lines can be extracted from an image. The scene elements whose edges don't check the planarity criteria are segmented as obstacles. The expression of these remaining edges in the IPM view allow a coarse segmentation of the obstacles location in front of the vehicle.

The described methodology have to be improved to perform an estimation of the free-space. The stereo-vision system has to be calibrated to represent the edges in a polar chart and the region free from obstacles has to be unified and precisely segmented from the bottom image to the intersection of the obstacles with the road plane.

\section{ACKNOWLEDGMENTS}

The author would like to thank Akihito Seki from the Okotumi Lab. for the interesting exchanges of these last months. This work is supported by the E.C. CyberCars ${ }^{2}$ project.

\section{REFERENCES}

[1] S. Sun et al., "On-road vehicle detection: a review", IEEE Transactions on Pattern Analysis and Machine Intelligence, vol. 28, $\mathrm{n}^{\circ}$ 5, may 2006, pp. 694-711.

[2] B. Steux et al., "FADE: a vehicle detection and tracking system featuring monocular vision and radar data fusion", IEEE Intelligent Vehicle Symposium (IV'02), Versailles, France, June 2002, pp. 632639.

[3] T. Rabie et al., "Active-Vision based traffic surveillance and control", Vision Interface Annual Conference (VI'01), Toronto, Canada, June 2001.

[4] R. Okada and K. Onoguchi, "Obstacle Detection Using Projective Invariant and Vanishing Lines", 9th IEEE International Conference on Computer Vision (ICCV'03), Nice, France, Oct. 2003.

[5] G.P. Stein and A. Shashua, "A robust method for computing vehicle ego-motion”, IEEE Intelligent Vehicle Symposium (IV'O0), Piscataway, NJ, USA, Oct. 2000.

[6] B. Heisele, "Motion-based object detection and tracking in color images sequences", Fourth Asian Conference on Computer Vision (ACCV'O0), Taipei, China, Jan. 2000, pp 1028-1033.

[7] C. Braillon et al., "Real-time moving obstacle detection using optical flow models", IEEE Intelligent Vehicle Symposium (IV'00), Tokyo, Japan, June 2006, pp 466-471.

[8] R. Labayrade et al., "Real time obstacle detection in stereo-vision on non flat road geometry through "v-disparity" representation", IEEE Intelligent Vehicle Symposium (IV'02), Versailles, France, Oct. 2002, pp 646-651.

[9] Z. Hu et al., "A Complete U-V-Disparity Study for Stereovision Based 3D Driving Environment Analysis", Fifth International Conference on 3-D Digital Imaging and Modeling (3DIM '05), Washington, DC, USA, 2005, pp. 204-211.

[10] M. Bertozzi et al., "Stereo Vision-based approaches for Pedestrian Detection", IEEE Computer Society Conference on Computer Vision and Pattern Recognition (CVPR'05), San Diego, CA, USA, June 2005, pp. $16-22$

[11] A. Broggi et al., "A Single Frame Stereo Vision System for Reliable Obstacle Detection during Darpa Grand Challenge 2005", IEEE Intelligent Transportation Systems Conference (ITSC'06), Toronto, Canada, June 2006, pp. 745-752.

[12] N. Simond and P. Rives, "Homography from a Vanishing Point in Urban Scenes", IEEE RSJ/International conference on Intelligent Robot and System (IROS'03), Las Vegas, Nev., USA, Oct. 2003.

[13] M. Okutomi et al., "Robust Estimation of Planar Regions for Visual Navigation Using sequential Stereo Images", IEEE International Conference on Robotics and Automation (ICRA'02), Washington DC, USA, May 2002, pp 3321-3327.

[14] H. A. Mallot et al., "Inverse perspective mapping simplifies optical flow computation and obstacle detection", Biological Cybernetics, Jan. 1991, pp. $177-185$.

[15] N. Simond and C. Laurgeau, "Vehicle Trajectory from an Uncalibrated Stereo-Rig with Super-Homography", IEEE RSJ/International conference on Intelligent Robot and System (IROS'06), Beijing, China, Oct. 2006 